\title{
Ad hoc networks capacity scaling problem
}

\author{
Adisa Hasković Džubur, Alem Čolaković, Belma Memić, Elma Avdagić-Golub \\ University of Sarajevo, Zmaja od Bosne 8, Sarajevo 71000, Bosnia and Herzegovina
}

\begin{abstract}
A large number of researchers have found interest in addressing the issue of capacity scaling for wireless ad hoc networks. This paper aims to provide a comprehensive overview of the development of capacity scaling laws in wireless networks, highlighting the problem of scaling as one of the basic challenges in their research. The review began with an analysis of ad hoc networks, where the possibility of their improvement was identified. After defining of the throughput capacity for random networks, which are taken as a reference model of consideration, there are also identified more advanced strategies for improving their throughput capacity. Based on these strategies, other factors that have an impact on capacity scaling laws have been identified and elaborated. In addition to the capacity of pure ad hoc networks, the capacity of hybrid wireless networks, i.e. networks in which at least two types of nodes functionally exist (ad hoc nodes/infrastructure nodes / auxiliary nodes), was performed and partially analyzed in this paper.
\end{abstract}

Keywords: capacity, scaling, ad hoc network

\section{Introduction}

Ad hoc networks are an increasingly relevant area of research due to their growing role and applicability in the development solutions of today's telecommunications. Continuous development of technology enables the application of mobile communication devices in almost all areas of human activity, such as medicine, transport, education, research, military applications, rescue missions, etc. Conceptually, mobile ad hoc networks represent the future of telecommunications, as they can operate quite self-sufficient of the existence of a fixed infrastructure. However, these networks still have a pronounced range of weaknesses, such as unresolved issues in terms of capacity, security and reliability. Therefore, it can be expected that in the coming years these networks will continue to be the subject of numerous researches.

The laws of capacity scaling of wireless networks provide a thorough understanding of how capacity scaling per node is performed in asymptotically large networks. The research was started in [1] where the analysis of the capacity of wireless networks was performed. Scaling of the space was performed, assuming that $\mathrm{n}$ nodes were distributed in the space of $1 \mathrm{~m}^{2}$. Each node can transmit $\mathrm{W}$ bits per second, via a common wireless channel. Two models that describe successful transmission over a single hop: protocol model and physical model; and two notions of network capacity were introduced: transport capacity and throughput capacity.

It is important to note that the notion of bandwidth differs from the notion of information-theoretical capacity, which describes the exact space of simulated communication speeds from a large number of senders to a large number of recipients, in the presence of interference and noise [2].

Capacity throughput has been studied asymptotically as the set of nodes continues to grow. The scaling result for random networks is pessimistic, because the flow per node $\frac{1}{\sqrt{n \log n}}$ weighs zero, when the set of nodes weighs infinity, which indicates that for fixed wireless networks of large dimensions. The main reason for such devastating data is that each node in the network shares a certain part of the channel's resources, geographical area, with other nodes in its immediate vicinity, which ultimately reduces their capacity. Simultaneous transmissions in a wireless network limit its bandwidth, which is caused by the presence of interference.

\section{Ad hoc networks}

An ad hoc network is a combination of more than two device able to communicate wirelessly and network. The 
term ad hoc networks today means wireless networks in which nodes communicate with each other by radio signal. They differ from classic mobile and wireless networks (WiFi, GSM, 3G, LTE) in that they do not rely on fixed infrastructure such as hot-spot antennas or base stations. Nodes in ad hoc networks have at the same time a data transfer (routing) function that takes place bidirectionally towards other nodes.

Wireless ad hoc networks can be sensor networks (Wireless Sensor Networks - WSN), vehicle networks (Vehicular Ad hoc Network - VANET), mesh networks, and the like. They have various purposes by nature, and their topologies depend on the specific application. Since they do not have a fixed routing infrastructure, it is necessary that they functionally have self-organizing mechanisms, which serve these purposes.[3]

Table 1. The concept of ad hoc networks according to standardization bodies

\begin{tabular}{cc}
\hline IEEE & IETF \\
\hline Ad hoc networks & $\begin{array}{c}\text { Mobile ad hoc networks } \\
\text { (MANET) }\end{array}$ \\
IEEE 802.11 standard: ad hoc - & IETF RFC 2501 \\
IBSS & \\
Single hop communication & Multihop communication \\
Physical and data layers & Network layer \\
\hline
\end{tabular}

Based on the characteristics of the nodes, ad hoc networks can be divided into homogeneous networks (all devices are identical, have the same characteristics and capabilities) and heterogeneous networks (devices are not identical, nor do they have the same capabilities). If the nodes are mobile, then these networks are referred to as mobile ad hoc networks (MANET), which will be the subject of this paper. [3]

\subsection{Mobile ad hoc networks}

Mobile ad hoc networks are dynamically formed structures, created using an autonomous system of mobile nodes, which are connected wirelessly without the use of existing network infrastructure, or centralized administration. Nodes have the freedom of random movement and arbitrary organization, and accordingly, the wireless network topology can change very quickly, without any possibility of prediction. Such a network may operate independently or may be connected to the Internet. Mobile ad hoc networks, in their operation, do not need the support of any form of fixed infrastructure, such as base stations. In principle, routes between nodes in an ad hoc network can be realized through multiple jumps, and it is therefore convenient to call such networks "multihop wireless ad hoc networks". Fig. 1 shows an example of mobile ad hoc networks and their communication topology.

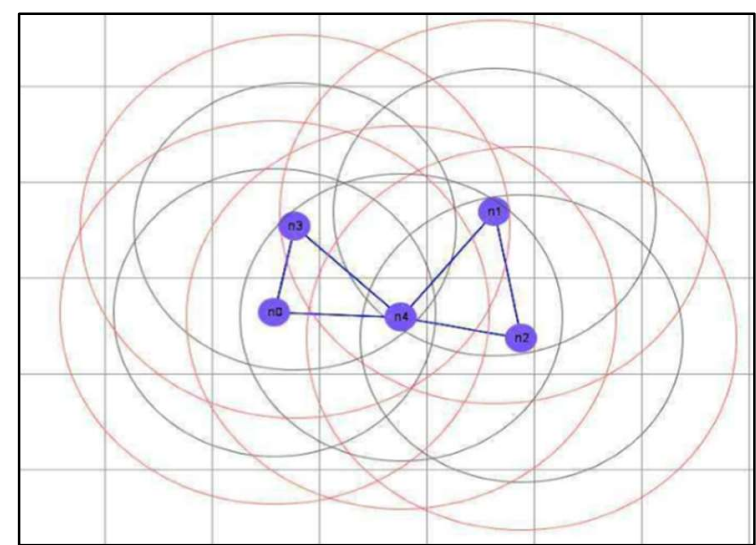

Figure 1. Topology of mobile ad hoc networks with marked transmission and interference band

Each node can communicate directly with other nodes, which are in the common transmission range. To communicate with nodes that are outside this range, the node should use proxy nodes to transmit jump-by-jump messages. [3]

The basic characteristics of MANET networks, whether inherited from wireless networks or specific only to ad hoc networks, can be described as follows:

1. Wireless - Nodes communicate wirelessly, thus sharing the same medium (radio, infrared, etc.).

2. Ad-hoc-based - Mobile ad hoc network is a current network, formed dynamically in an arbitrary way, by collecting nodes at a given time and according to the indicated need.

3. Autonomous and without infrastructure - MANET does not depend on any established infrastructure or centralized administration. Each node operates in a distributed peer-to-peer mode, as an independent router and generates independent data.

4. Multihop routing - dedicated routers are not required here. All nodes act as routers and forward packets to each other to allow the mutual exchange of information.

5. Mobility - Each node has freedom of movement when communicating with other nodes. The topology of such an ad hoc network is dynamic due to the constant movement of its nodes.

\subsection{Space to improve ad hoc networks}

The ad hoc network has been a popular field of research over the last few years. Almost every aspect of 
the network has been explored to some level of detail. However, in the end, the solution to none of the problems was completely found. On the contrary, several questions arose. Similar to ad hoc networks, many aspects of sensor networks have also been explored, but, unlike ad hoc networks, there are many more issues to be addressed within this framework. Topics to be addressed in ad hoc networks are [4]:
1. Scalability
2. Quality of service
3. Client-server model
4. Security
5. Interoperability with the Internet
6. Energy conservation.

Scalability - Most visionaries who describe applications using ad hoc and sensor network technologies take network scalability lightly. Imagine, for example, a network that can be "any size". It becomes unclear how one such network can grow? Ad hoc networks have a problem of capacity scalability. As an example, take simple research in the field of interference. In a non-cooperative network, where omnidirectional antennas are used, the permeability per node decreases at a rate of $\frac{1}{\sqrt{N}}$, where $\mathrm{N}$ is the number of nodes. This means that in a network with 100 nodes, one node gets approximately $1 / 10$ of the theoretical network speed. This problem is solved by improvements on the physical layer, such as the use of directional antennas. If the available capacity sets certain limitations in communication, this is immediately reflected in the protocols as well. Route information, location services, and encryption key exchange are just some examples of problems that will require significant consideration, in a situation of increasing network nodes. If limited resources were spent on intensive control traffic, these networks might never come to life. In this regard, scaling is taken as an extremely important field of research, and as such must be treated in the design of solutions for ad hoc and sensor networks. [4]

Quality of service - The heterogeneity of existing internet applications poses a challenge to network designers who have built the network to provide only the best-effort service. QoS must be guaranteed by the network, which should ensure performance for particular traffic, in terms of QoS parameters such as delay, jitter, bandwidth, probability of packet loss, etc. Regardless of previous research efforts in the QoS area, QoS in ad hoc and sensor networks still represents an unexplored area. The issues of QoS robustness, QoS routing policies, algorithms, and protocols with multiple priorities should continue to be addressed. [4]

Client-server model - The Internet network client is generally configured to use the server as its "partner" for all network activities. On the other hand, the structure in ad hoc networks cannot be defined by collecting IP addresses in subnets. There may not be a server, but the demand for basic services still exists. Address allocation, name resolution, authentication, and location service are just examples of basic services that are necessary for the network, but their position in the network is unknown, and perhaps time-varying. Due to the non-infrastructural nature of these networks and the mobility of their nodes, a different approach to solving this problem needs to be applied. It is still unclear who will be responsible for managing the various network services. Therefore, although there have been many research initiatives in this area, the issue of transition from the traditional clientserver model remains to be adequately addressed. [4]

Security - Ad hoc and sensor networks are particularly prone to malicious behavior. The lack of any form of centralized network management makes these dynamic wireless structures extremely vulnerable to infiltrations, eavesdropping, interference, etc. Security is indeed one of the most difficult problems to solve, but has received only modest attention so far. The "golden age" of this area of research can be expected only after solving functional problems at lower strata. [4]

Internet Interoperability - The issue of defining the interface between two very different networks is not simple. If a node has an Internet connection, it can offer an Internet connection to other nodes. This node can declare itself the default router, and the entire network can be viewed as a "single-hop" from an Internet perspective. [4]

Energy conservation - Energy conservation networks are becoming extremely popular in ad hoc research communities, especially sensor networks. Energy conservation is currently being discussed at all layers of the stack protocol. Two basic research topics that are almost identical: maximizing the life of one battery and maximizing the life of the entire network. These goals can be achieved either by developing better batteries or by building more energy-efficient network terminals. Energy efficiency at the network interface can be improved by developing technology transmission/reception. [4]

\subsection{Ad hoc network Throughput Capacity}

Assuming that all nodes of the random ad hoc network are homogeneous, ie all transmissions use the same nominal power range, models that enable secure transmission will be defined first: protocol model and physical model. [1]

1. Protocol model: all nodes use the same range $r$ for all transmissions. When node $X_{i}$ transmits to node $X_{j}$ via the m-th subchannel, this transmission is successful if: 
a. The distance between $X_{i}$ and $X_{j}$ is not greater than $r$, i.e. $\left|X_{i}-X_{j}\right| \leq r$.

b. For every other node $X_{k}$ that simultaneously transmits over the same subchannel, the following holds $\left|X_{k}-X_{j}\right| \leq(1+\Delta) r$.

2. Physical model: All nodes choose a common power level $P$ for all their transmissions. Let $\left\{X_{k} ; k \in \mathcal{T}\right\}$ be a subset of nodes that simultaneously transmit at some point in time by a given subchannel. The transfer from node $X_{i}$, $i \in \mathcal{T}$, was successfully received at node $X_{j}$ if [1]:

$$
\frac{\frac{P}{\left|X_{i}-X_{j}\right|^{\alpha}}}{N+\sum_{\substack{k \in \mathcal{T} \\ k \neq i} \frac{P}{\left|X_{k}-X_{j}\right|^{\alpha}}}} \geq \beta
$$

where $\beta$ is the minimum value of the signalinterference ratio (SIR) required for successful transmission, and $\alpha>2$.

Definition of throughput capacity: Let $N$ be the total number of nodes in the network. Denote by $\lambda(N)$ the flow for each node, which is defined as the average baud rate, measured in bits or packets per unit time, which can be uniformly valid for all nodes in the network, when communicating with the destination. This means that there is $T<\infty$, such that in each time interval $[(i-1) T$, $i T]$ each node can send $T \lambda(N)$ bits to its corresponding destination. [1]

Definition of Feasible Throughput: The flow per node of $\lambda(N)$ bits / second is said to be feasible if there is a spatial and temporal scheme for sending schedules, such that each node can send an average of $\lambda(N)$ bits / second its destination node.

Definition of the random wireless networks throughput capacity: The bandwidth of random wireless networks has the form $\Theta(f(N))$ bits/seconds, if there are deterministic constants $c_{1}>0$ and $c_{2}<\infty$ such that [1]:

$$
\begin{gathered}
\lim _{N \rightarrow \infty} \operatorname{Pr}\left(\lambda(N)=c_{1} f(N) \text { is feasible }\right)=1 \\
\lim _{N \rightarrow \infty} \inf \operatorname{Pr}\left(\lambda(N)=c_{2} f(N) \text { is feasible }\right)<1
\end{gathered}
$$

In [1] it was found that by applying a protocol model in an interference-free environment, the capacity of wireless networks with $\mathrm{n}$ randomly arranged nodes, each being able to transmit $\mathrm{W}$ bits per second, using the same range as randomly selected (and thus probably in more remote ) destination is given as in Eq. (3):

$$
\lambda(N)=\Theta\left(\frac{W}{\sqrt{n \log n}}\right)
$$

This is the case if the nodes are located on the surface of a three-dimensional sphere or the surface of a flat disk. Even when the nodes are optimally located on the disk per unit area, and the rank for each transmission is optimally selected, the wireless network cannot achieve a flow to any node of a distance of $1 \mathrm{~m}$ greater than $\Theta\left(\frac{W}{\sqrt{n}}\right)$ bits per second. Ultimately, a wireless network on a unit surface disk cannot transmit more than $\Theta(W \sqrt{n})$ bitmeters/second, regardless of how the load is distributed. In the presence of a physical model without interference, the lower flow limits are the same as in the protocol model, while the upper flow limits are $\Theta\left(\frac{W}{\sqrt{n}}\right)$ for random networks and $\Theta\left(\frac{W}{n \frac{1}{\alpha}}\right)$ for arbitrarily selected networks. The division of channels into several subchannels does not affect these claims.

\subsection{Strategies to improve throughput capacity}

Reflections on possible throughput capacity improvements of random networks by introducing more advanced techniques and more sophisticated strategies, made that following research based on capacity scaling, have contributed to a positive outcome.

Initially, it was observed that by enabling transmission, both over long and short distances, the throughput can be slightly improved with $\Theta\left(\frac{1}{\sqrt{n}}\right)$ [5]. The scheme developed to achieve this flow was implemented on multi-hop transmission, paired encoding and decoding of each hop, and a multiple time allocation approach. Throughput capacity improvement can also be achieved by appropriate antenna routing, with several patterns described in [4] showing that bandwidth can be improved with a gain of $\frac{4 \pi^{2}}{\alpha \beta}$ using directional transmissions and receptions, where $\alpha$ and $\beta$ are antenna parameters. Furthermore, in the research described in [6], it was obtained that the maximum gain of the value of the value $\Theta\left((\log n)^{2}\right)$ using the direction of the antennas on the transmitters and receivers, which corresponds to the flow $\Theta\left(\frac{(\log n)^{3 / 2}}{\sqrt{n}}\right)$. If the nodes have the ability to receive multiple packets of MPR (Multi-Packet Reception), where the receiver can decode the received packets, an increase in capacity per node can be achieved. For the same conditions, the MPR throughput capacity of random ad hoc networks can be improved by at least an order of magnitude $\Theta(\log \mathrm{n})$ for the protocol model and $\Theta\left((\log n)^{\frac{\alpha-2}{2 \alpha}}\right)$ for the physical model, at where $\alpha$ is the exponent that determines the transmission losses in the physical model, which is shown in [7]. If long-distance 
communication is present, with multiple inputs and multiple outputs (MIMOs) that are in cooperation, in [8] significant progress has been made in determining throughput, and capacity scaling has been performed for almost constant value per node to $\Theta\left(n^{-\epsilon}\right)$, where $\epsilon>0$ can be arbitrarily small.

However, the gain in increasing capacity also depends on the complexity of the system, which arises due to intelligent hierarchical cooperation between nodes. Regardless of the complexity of the constructed strategy, the result in [9] is inspiring but still controversial. Franceschetti in [10] argues that throughput capacity greater than $\Theta\left((\log N)^{2} / \sqrt{\mathrm{N}}\right)$ cannot be achieved due to the limitation of the degree of freedom resulting from the laws of physics. Certain assumptions and models have led to claims of impossible linear capacity scaling in [10]. In response, on the other hand, using Maxwell's equations, Lee in [11] establishes the laws of scaling capacities in visible space (LOS - line-of-sight), which show that linear scaling of total (aggregate) throughput for stationary ones is indeed possible. random networks. It is important to note that even if there are physical boundaries of this type, sophisticated strategies such as hierarchical collaboration cannot further improve node throughput described by $\Theta(1 / \sqrt{\mathrm{N}})$, in terms of throughput capacity scaling, these strategies can generally be useful in networks of any finite dimensions.

These studies consider networks assuming that network bandwidth is limited and that each node can transmit $W \frac{b i t}{s}$. On the other hand, it is interesting to observe a scenario in which nodes have limited power, but unlimited bandwidth. Several studies have been done on the topic of ultra-broadband techniques, and finally, in [12] it was shown that ad hoc networks throughput capacity in which the power of nodes is limited and the range is unlimited, is scaled with $\Theta\left(P_{0} N^{(\alpha-1) / 2}\right)$.

Without relying on the mentioned advanced techniques in fixed random networks, the treatment of ad hoc networks problems begins in case if their nodes are mobile. The influence of node mobility on capacity scaling was first treated in [13]. By applying the mobility model to each node, it is shown that throughput of the mobile ad hoc network remains constant $\Theta(1)$, using a two-jump scheme between adjacent relays and creates a final but unknown (arbitrary) delay. This leaves a significant impact on network throughput, as it leads to more efficient capacity utilization. If we analyze direct transmission (Figure 3) interference occurs between distant nodes, or equivalently, a spatially large area is required for such communication, and thus the number of simultaneous transmissions is reduced. On the other hand, if the network only allows communication between the nearest neighbors (Figure 4), most packets will be delivered through multiple hops (Figure 5), leading to a reduction in the use of available capacity. The following figures illustrate packet transmission strategies in mobile ad hoc networks:

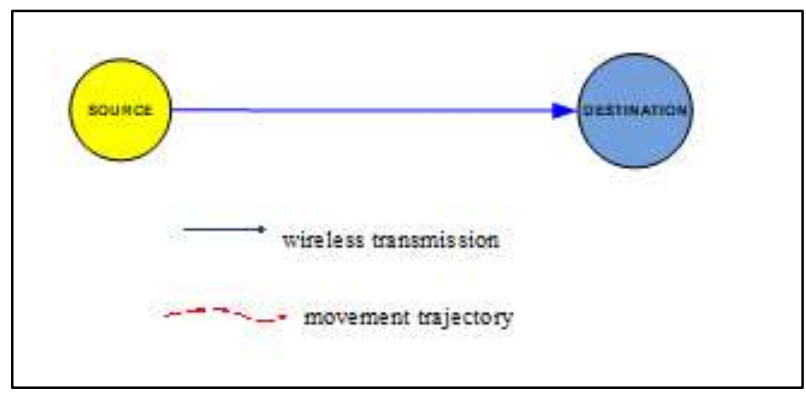

Figure 3. Direct communication

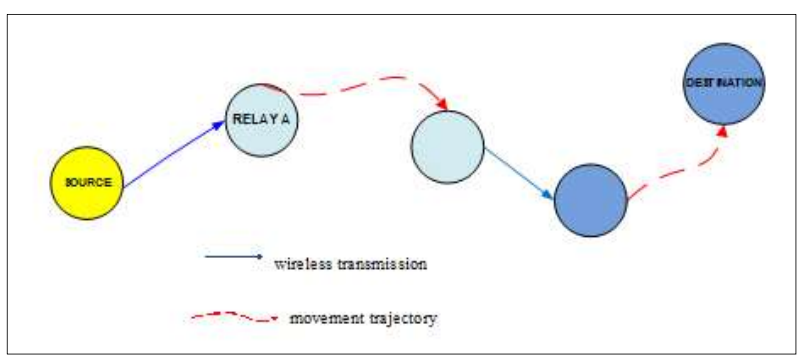

Figure 4. Two-hop communication in a mobile ad hoc network

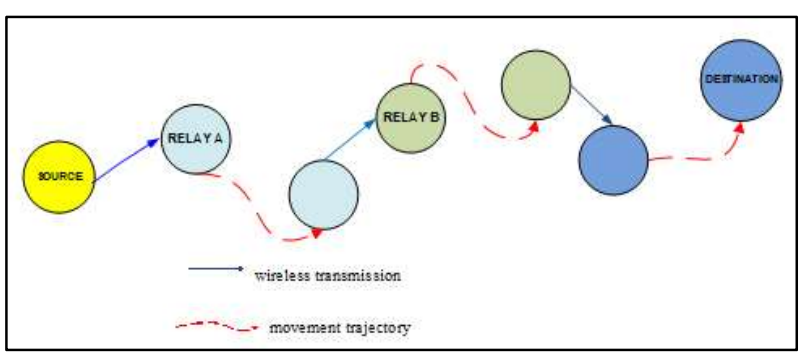

Figure 5. Multi-hop communication in a mobile ad hoc network

\subsection{The capacity of infrastructure supported ad hoc networks}

It has been shown that the addition of wired infrastructure to nodes, such as base stations, in ad hoc networks can make significant advantages in the context of power, capacity, and delay parameters. Fixed infrastructure supports ad hoc networks by transmitting their packets, not just representing access points to the Internet. The advantage of infrastructure nodes is in overcoming geographical limitations since packets can be transmitted over long distances over a high-bandwidth wired connection, as a supplement (assistance) to local ad hoc transmissions. 
A study on the capacity scaling of hybrid wireless networks was performed in [14]. By setting $N$ stationary nodes and $M$ base stations in the network, it is shown that the throughput increases linearly with $M$, if $M=$ $\omega(\sqrt{N})$, otherwise the improvement is negligible.

In contrast to the hexagonal base cell structure in [34], the access points in [15] are randomly distributed in the network and the results show that it is possible to achieve a bandwidth of $\left(\frac{1}{\log N}\right)$ provided that the number of ad hoc nodes is connected with each access point limited by the upper value. Assuming that random access point assignments are performed, as well as the presence of a general channel fading model, capacity limits of hybrid wireless networks similar to those previously described are obtained.

Somewhat later, in the literature [16], the shape of the upper bandwidth limits for a single node was obtained, in a network with randomly arranged ad hoc nodes, which communicate with each other through common wireless connections of $W$ bita/s capacity and base stations set in any deterministic form. By enabling control of the power of base stations, three regimes of asymptotic scaling of bandwidth per user were determined at the same time, depending on the increase in the number of infrastructure nodes $M$ concerning to the number of ad hoc nodes $N$.

Table 2. Scaling modes [16]

\begin{tabular}{crr}
\hline $\begin{array}{c}\text { Throughput } \\
\text { capacity } \\
\text { scaling mode }\end{array}$ & $\begin{array}{c}\text { The ratio of the } \\
\text { number of infrastructure } \\
\text { and ad hoc mobile nodes }\end{array}$ & $\begin{array}{l}\text { Throughput } \\
\text { capacity per } \\
\text { user }\end{array}$ \\
\hline 1. & $M \lesssim \sqrt{N / \log N}$ & $\Theta\left(\frac{W}{\sqrt{N \log N}}\right)$ \\
2. & $\sqrt{N / \log N \lesssim M \lesssim N / \log N}$ & $\Theta\left(\frac{W M}{N}\right)$ \\
3. & $M \gtrsim N / \log N$ & $\Theta\left(\frac{W}{\log N}\right)$ \\
\hline
\end{tabular}

\subsection{Case study}

An illustrative representation of the results from the previous table can be seen in the following graph. The graph on Figure 6 shows the application of the scaling mode according to the network conditions in terms of the number of nodes (mobile and infrastructure). The total number of observed infrastructure nodes is 5, while the number of mobile nodes was changed in ten iterations (from 100 to 200), and accordingly, the corresponding values for throughput were obtained (flowing the application of the conditions of a certain scaling mode).
Having in mind the set values for $M$ and $N$, it is noticed that the combination of bandwidth of 1 st and 2 nd mode gives the corresponding results. Mode 3, in this case, gives the highest bandwidth values, but due to the set value of $M$, which is less than $(N / \log N)$ in each observed iteration, as well as the fact that the bandwidth expression defined for this mode is not in functions of the number of infrastructure nodes, the same cannot be treated as relevant.

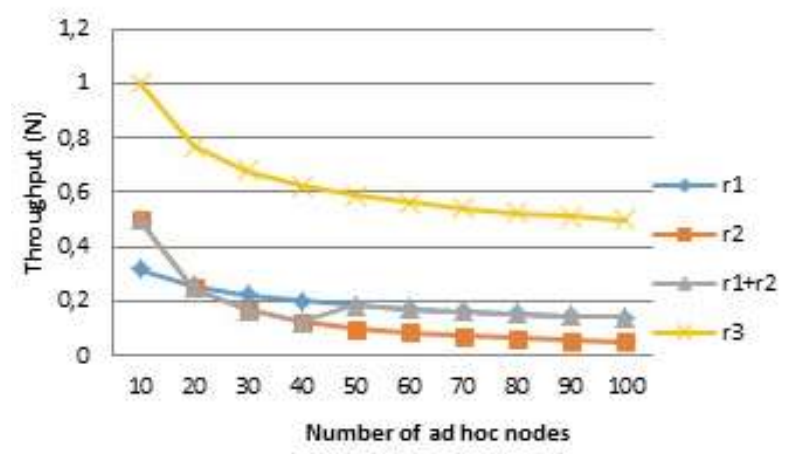

Figure 6. Example of application of scaling mode

It can be seen that there is no need to implement any form of infrastructure for regime 1, given that bandwidth is achievable with the simple use of ad hoc communication; and for regime 3, the addition of additional infrastructure nodes does not make any improvement in throughput, at least not in an appropriate sense.

Noting that previous studies have most commonly considered a two-dimensional square or disk-shaped network area, further research has moved toward determining the influence of network area geometry on capacity scaling by exploring one-dimensional and twodimensional networks with regularly placed base stations.

The main implications of their results (shown in Table 3) are: (i) for one-dimensional networks, even a small number of base stations added can significantly increase the throughput for a single node; and (ii) for twodimensional networks of a rectangular structure, the capacity scaling behavior is the same as for a onedimensional network or a two-dimensional square-shaped network, depending on its area. The influences of both the network topology and the traffic pattern, which differ according to the number of destination nodes, are considered.

Table 3. Influence of network geometry on throughput capacity scaling [17]

\begin{tabular}{ccc}
$\begin{array}{c}\text { The geometric } \\
\text { shape area } \\
\text { network }\end{array}$ & $\begin{array}{c}\text { Number of base } \\
\text { stations }\end{array}$ & $\begin{array}{c}\text { Capacity } \\
\text { throughput }\end{array}$ \\
\hline
\end{tabular}




\begin{tabular}{|c|c|c|}
\hline One-dimensional grid & $M \log M=O(N)$ & $\Omega(M / N)$ \\
\hline $\begin{array}{l}\text { Two-dimensional } \\
\text { rectangle grid width } \\
o(\log N)\end{array}$ & $M \log M=\omega(N)$ & $\Omega(1 / \log M)$ \\
\hline $\begin{array}{l}\text { Two-dimensional } \\
\text { square grid }\end{array}$ & $M=O(\sqrt{N})$ & $\Omega(1 / \sqrt{N})$ \\
\hline $\begin{array}{l}\text { Two-dimensional } \\
\text { square grid } \Omega(\log N)\end{array}$ & $M=\omega(\sqrt{N})$ & $\begin{array}{l}\Omega(\min \{M \\
/ N, 1 \\
/ \log M\})\end{array}$ \\
\hline
\end{tabular}

An important implication of the mentioned results is in the conclusion that the capacity gain is insignificant if the number of infrastructure nodes distributed in the network area of the geometric shape of a square or disk grows asymptotically slower than a certain threshold. Emphasizing that such a "threshold" comes from the insufficient degree of utilization of the base station's capabilities, leads to a more efficient scaling of the capacity. The basic strategy of this form is reflected in the delivery of packets to the nearest base station through multiple hops, as opposed to single-hop transmission from the node to the corresponding base station, which results in sublinear scaling of capacity with the number of base stations. Consideration of the problem of capacity scaling in hybrid wireless networks went in the direction of exploiting the routing strategy of $\mathrm{L}$ maximum jumps. Namely, if the destination can be reached for L jumps, source packets are delivered without relying on any form of infrastructure nodes. More importantly, it has been shown that without throughput disruption, network latency can be significantly improved, however, to the detriment of infrastructure construction. At the same time, it is possible to achieve a constant value of throughput and latency in this type of network.

Continuation of research in this area was in the treatment of the impact of directional antennas and their implementation on base stations, as well as consideration of the impact of multi-antenna systems on single base station on network throughput capacity.

\section{Conclusion}

In recent years, there is increasing usability and implementation of wireless networks. This increases the functional requirements of the network, as well as the density of nodes in the network. The increased dimensions of the network, develop many problems that require a separate approach to solving. The basic problems in ad hoc networks, which are highlighted in this paper are scalability, service quality, client-server model, security, interoperability with the Internet, energy conservation, node interoperability, while the problem of scaling has been predominantly treated..

The laws of capacity scaling of wireless networks provide a thorough understanding of how capacity scaling is performed per node, in asymptotically large networks. The paper reviews and critically analyzes previous research on the problem of wireless network capacity with special reference to ad hoc networks. Based on this review and analysis, the classification of the applicability of individual approaches to the problem of network scaling was performed. The dynamics that ad hoc networks bring with them develop the need to examine and theoretically understand the scaling limits of these networks.

In addition to the capacity of pure ad hoc networks, the paper provides an overview of research on the capacity of hybrid networks. This research aims to determine the possibility of scaling networks with heterogeneous nodes and to compare the obtained scaled throughput capacity values, with the values achieved by applying throughput capacity improvement strategies, in pure ad hoc networks.

\section{References}

[1] P. Gupta and P. R. Kumar, "The capacity of wireless networks," IEEE Trans. Inf. Theory, 2000.

[2] T. M. Cover and J. A. Thomas, Elements of Information Theory. 1991.

[3] S. Basagni, M. Conti, S. Giordano, and I. Stojmenovic, Mobile ad hoc networking. 2005.

[4] C. de M. Cordeiro and D. P. Agrawal, Ad hoc and sensor networks: Theory and applications (2nd edition). 2011.

[5] M. Franceschetti, O. Dousse, D. N. C. Tse, and P. Thiran, "Closing the gap in the capacity of wireless networks via percolation theory," IEEE Trans. Inf. Theory, 2007.

[6] C. Peraki and S. D. Servetto, "On the maximum stable throughput problem in random networks with directional antennas," in Proceedings of the International Symposium on Mobile Ad Hoc Networking and Computing (MobiHoc), 2003.

[7] H. R. Sadjadpour, Z. Wang, and J. J. Garcia-LunaAceves, "The capacity of wireless ad hoc networks with multi-packet reception," IEEE Trans. Commun., 2010.

[8] S. Aeron and V. Saligrama, "Wireless ad hoc networks: Strategies and scaling laws for the fixed SNR regime," IEEE Trans. Inf. Theory, 2007.

[9] A. Özgür, O. Lévêque, and D. N. C. Tse, "Hierarchical cooperation achieves optimal capacity scaling in $\mathrm{Ad}$ Hoc networks," IEEE Trans. Inf. Theory, 2007.

[10] M. Franceschetti, M. D. Migliore, and P. Minero, "The capacity of wireless networks: Information-theoretic and physical limits," IEEE Trans. Inf. Theory, 2009. 
[11] S. H. Lee and S. Y. Chung, "Capacity scaling of wireless ad hoc networks: Shannon meets maxwell," IEEE Trans. Inf. Theory, 2012.

[12] H. Zhang and J. C. Hou, "Capacity of wireless ad-hoc networks under Ultra Wide Band with power constraint," in Proceedings - IEEE INFOCOM, 2005.

[13] M. Grossglauser and D. N. C. Tse, "Mobility increases the capacity of ad hoc wireless networks," IEEE/ACM Trans. Netw., 2002.

[14] B. Liu, Z. Liu, and D. Towsley, "On the capacity of hybrid wireless networks," in Proceedings - IEEE INFOCOM, 2003.

[15] U. C. Kozat and L. Tassiulas, "Throughput Capacity of Random Ad Hoc Networks with Infrastructure Support," in Proceedings of the Annual International Conference on Mobile Computing and Networking, MOBICOM, 2003.

[16] A. Zemlianov and G. De Veciana, "Capacity of ad hoc wireless networks with infrastructure support," IEEE J. Sel. Areas Commun., 2005.

[17] B. Liu, P. Thiran, and D. Towsley, "Capacity of a wireless ad hoc network with infrastructure," in Proceedings of the International Symposium on Mobile Ad Hoc Networking and Computing (MobiHoc), 2007. 